\title{
The role of glycaemic and lipid risk factors in mediating the effect of BMI on coronary heart disease: a two-step, two-sample Mendelian randomisation study
}

\author{
Lin $\mathrm{Xu}^{1,2,3}$ • Maria Carolina Borges ${ }^{2,4}$ - Gibran Hemani ${ }^{2,5}$ - Debbie A. Lawlor ${ }^{2,5}$
}

Received: 27 March 2017 / Accepted: 29 June 2017 / Published online: 9 September 2017

(C) The Author(s) 2017. This article is an open access publication

\begin{abstract}
Aims/hypothesis The extent to which effects of BMI on CHD are mediated by glycaemic and lipid risk factors is unclear. In this study we examined the effects of these traits using genetic evidence.

Methods We used two-sample Mendelian randomisation to determine: (1) the causal effect of BMI on CHD $(60,801$ case vs 123,504 control participants), type 2 diabetes ( 34,840 case vs 114,981 control participants), fasting glucose $(n=46,186)$, insulin $(n=38,238), \mathrm{HbA}_{1 \mathrm{c}}(n=46,368)$ and LDL-cholesterol, HDL-cholesterol and triacylglycerols $(n=188,577)$; (2) the causal effects of glycaemic and lipids traits on CHD; and (3) the extent to which these traits mediate any effect of BMI on CHD. Results One SD higher BMI $\left(\sim 4.5 \mathrm{~kg} / \mathrm{m}^{2}\right)$ was associated with higher risk of CHD (OR 1.45 [95\% CI 1.27, 1.66]) and type 2 diabetes $(1.96$ [95\% CI 1.35, 2.83]), higher levels of fasting glucose $(0.07 \mathrm{mmol} / 1$ [ $95 \% \mathrm{CI} 0.03,0.11]), \mathrm{HbA}_{1 \mathrm{c}}$ $(0.05 \%$ [95\% CI $0.01,0.08])$, fasting insulin $(0.18 \log \mathrm{pmol} / 1$
\end{abstract}

Electronic supplementary material The online version of this article (doi:10.1007/s00125-017-4396-y) contains peer-reviewed but unedited supplementary material, which is available to authorised users.

Debbie A. Lawlor

d.a.lawlor@bristol.ac.uk

School of Public Health, Sun Yat-sen University, Guangzhou 510080, Guangdong, People's Republic of China

2 MRC Integrative Epidemiology Unit, University of Bristol, Rm OS11, Oakfield House, Oakfield Grove, Bristol BS8 2BN, UK

3 School of Public Health, University of Hong Kong, Hong Kong Special Administrative Region, People's Republic of China

4 Postgraduate Program in Epidemiology, Federal University of Pelotas, Pelotas, Brazil

5 School of Social and Community Medicine, University of Bristol, Bristol, UK
[95\% CI 0.14, 0.22]) and triacylglycerols $(0.20 \mathrm{SD}$ [95\% CI $0.14,0.26])$ and lower levels of HDL-cholesterol $(-0.23 \mathrm{SD}$ $[95 \% \mathrm{CI}-0.32,-0.15])$. There was no evidence for a causal relation between BMI and LDL-cholesterol. The causal associations of higher triacylglycerols, $\mathrm{HbA}_{1 \mathrm{c}}$ and diabetes risk with CHD risk remained after performing sensitivity analyses that considered different models of horizontal pleiotropy. The BMI-CHD effect reduced from 1.45 to 1.16 $(95 \%$ CI $0.99,1.36)$ and to $1.36(95 \%$ CI $1.19,1.57)$ with genetic adjustment for triacylglycerols or $\mathrm{HbA}_{1 \mathrm{c}}$, respectively, and to 1.09 (95\% CI 0.94, 1.27) with adjustment for both. Conclusions/interpretation Increased triacylglycerol levels and poor glycaemic control appear to mediate much of the effect of BMI on CHD.

Keywords Body mass index - Cardiovascular disease risk factors $\cdot$ Coronary heart disease $\cdot$ Mediation $\cdot$ Mendelian randomisation

$\begin{array}{ll}\begin{array}{l}\text { Abbreviations } \\ \text { CARDIoGRAM }\end{array} & \begin{array}{l}\text { Coronary ARtery DIsease Genome wide } \\ \text { Replication And Meta-analysis } \\ \text { DIAbetes Genetics Replication And } \\ \text { Deta-analysis } \\ \text { Genetic Investigation of ANthropometric }\end{array} \\ \text { GIANT } & \begin{array}{l}\text { Traits } \\ \text { Global lipids genetics consortium }\end{array} \\ \text { GLGC } & \begin{array}{l}\text { Genome-wide associations studies } \\ \text { GWverse variance weighted }\end{array} \\ \text { IVW } & \text { Meta-analyses of glucose and insulin- } \\ \text { MAGIC } & \text { related traits consortium } \\ \text { Mendelian randomisation }\end{array}$




\section{Introduction}

Greater BMI is a risk factor for a wide range of adverse health outcomes, including CHD the leading cause of death worldwide. Whilst preventing overweight and obesity is an important public health aim, the substantial and increasing number of people with a high BMI highlights the need for secondary prevention that aims to reduce risk of the main disease outcomes of high BMI, such as CHD, by targeting causal mediators. This is also important because beyond bariatric surgery there are no effective and sustainable treatments for those who are obese [1].

Large prospective population studies show that higher BMI is associated with adverse blood lipid levels, higher fasting glucose and insulin, type 2 diabetes mellitus and CHD. RCTs show that elevated triacylglycerols, LDL-cholesterol, glucose and BP increase the risk of CHD [2,3]. Thus, the association of BMI with CHD could be mediated by these established modifiable risk factors. However, the common method used to test for mediation, by observing how much the confounder-adjusted multivariable association between a risk factor (e.g. BMI) and outcome (e.g. CHD) reduces with further adjustment for potential mediators [4], has been shown to be biased in many situations [5].

Mendelian randomisation (MR), the use of genetic variants as instrumental variables to test the causal effect of risk factors on outcomes, is unlikely to be biased by the extensive confounders of multivariable observational analyses, is less prone to measurement error [6] and, because genetic variants are fixed at conception, cannot be biased by reverse causality $[7,8]$. As such, MR has been used increasingly over the past decade to provide more robust estimates for the causal effect of many risk factors on a range of health outcomes, with results from MR closely resembling those from RCTs where both are available (e.g. the effect of LDL-cholesterol [9] and systolic BP [10] on CHD). Recently, methods have been developed for its use in testing causal mediation using a two-step approach that is considerably less prone to the biases inherent in the common multivariable approach [5]. Figure 1 provides a brief description of MR and its assumptions.

Previous MR studies using data from three collections have shown that higher BMI causally relates to higher risk for CHD (the results of our meta-analysis of these previous MR studies are presented in the electronic supplementary material [ESM] Fig. 1) [11-14]. These studies used one-sample MR and were unable to undertake sensitivity analyses that have been developed for testing likely bias by pleiotropy [15]. The number of cases of CHD varied from 3062 to 11,056, which are modest for MR studies. Although MR is likely to be less biased than conventional multivariable approaches, it usually requires a considerably larger sample size. Only one of these MR studies analysed potential mediators of the impact of BMI on CHD. It concluded that LDL-cholesterol, remnant cholesterol and systolic BP, explained $8 \%, 7 \%$ and $7 \%$, respectively, of the effect of BMI on CHD [14]. That study was unable to explore potential mediation by insulin sensitivity or hyperglycaemia, which are strongly influenced by BMI and are strong risk factors for CHD. Here, we aimed to investigate the mediating effects of lipid and insulin/ glycaemic traits on the effect of BMI on CHD using a large MR study, including over 60,000 individuals with CHD, and to analyse a wider set of potential mediators including glycaemic traits (fasting glucose and insulin, $\mathrm{HbA}_{1 \mathrm{c}}$, type 2 diabetes) than previous studies.

\section{Methods}

We used two-step two-sample MR [5, 16] with publicly available datasets that provide genome-wide association results for BMI, glycaemic traits, lipids and CHD. Two-sample MR refers to the use of different datasets (samples) to obtain the gene-risk factor (e.g. BMI) and gene-outcome (e.g. CHD) associations. First, we tested the effects of BMI on CHD, and then the effects of potential mediation using two-step MR. In step one we tested causal effects of BMI on potential mediators and in step two the causal effects of potential mediators on CHD [5].

\section{Data sources}

Genetic instrumental variable for BMI From the most updated genome-wide associations studies (GWAS) on BMI, the Genetic Investigation of ANthropometric Traits (GIANT) consortium, we obtained 77 SNPs, identified from the primary meta-analysis of 322,154 European-descent individuals, independently contributing to BMI at genome-wide significance $\left(p<5 \times 10^{-8}\right)$ [17]. These variants were defined as being independent of each other on the basis of low correlation $\left(R^{2}<0.1\right)$ in HapMap22 or the 1000 Genome project data. These 77 SNPs account for $2.4 \%$ of BMI phenotypic variance [17]. For sensitivity analyses, we included 20 SNPs from the secondary analysis of this GWAS [17]; these include some SNPs that did not reach genome-wide significance in Europeans.

Potential mediators Associations of SNPs with the phenotypes were extracted from publicly available GWAS consortia. Data on type 2 diabetes mellitus GWAS correlates was obtained from the DIAbetes Genetics Replication And Meta-analysis (DIAGRAM, http://diagram-consortium.org/ downloads.html, accessed on 22 June 2016), which includes 34,840 case and 114,981 control participants of European origin [18]. Genetic associations with fasting insulin $(n=38,238)$, fasting glucose $(n=46,186)$ and $\mathrm{HbA}_{1 \mathrm{c}}$ 


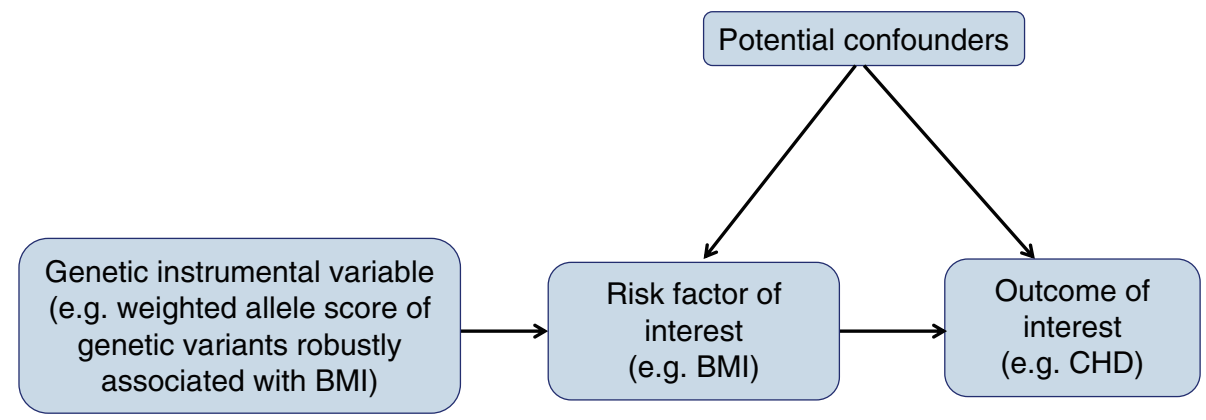

Fig. 1 Summary of MR and its assumptions. The underlying assumptions of MR are that: (1) the genetic instrumental variable(s) are robustly related to the risk factor of interest (here BMI; this is illustrated by the arrow from the genetic instruments to BMI); (2) there is no relationship between any confounders of the risk factor (BMI) and outcome (CHD) and the genetic instrumental variable (illustrated by the lack of any arrow between these confounders and the genetic instrument); and (3) there is no path from the genetic instrument to the outcome other than through its relationship to the risk factor (illustrated by the lack of any arrow that goes directly from the genetic instrument to the outcome). Empirical evidence suggests that the most likely of these three assumptions to be violated, and result in potentially biased results, is the last one. This may be violated in MR studies by horizontal pleiotropy (i.e. where the genetic instrument $[\mathrm{s}]$ affect other factors which, independent of their impact on the risk factor of interest, influence the outcome). If this horizontal pleiotropy is present then the MR estimate of the effect of a risk factor on outcome will be biased, it will actually be the combined effect of that risk factor and any other (pleiotropic) paths from the genetic instruments to outcome. The bias could be an exaggeration of the true

( $n=46,368)$ were obtained from the Meta-Analyses of Glucose and Insulin-related traits Consortium (MAGIC, http://www.magicinvestigators.org/, accessed on 22 June 2016); the participants were of European ancestry without diabetes [19]. Genetic associations with HDLcholesterol, LDL-cholesterol and triacylglycerols in 188,577 Europeans were obtained from the Global Lipids Genetics Consortium (GLGC) investigators (http://csg.sph.umich.edu/ abecasis/public/lipids2013/, accessed on 22 June 2016) [20].

Study outcome: CHD Data on coronary artery disease/ myocardial infarction were obtained from the Coronary ARtery DIsease Genome wide Replication And Meta-analysis (CARDIoGRAM) plusC4D investigators (www.CARDIOGRAMPLUSC4D.ORG, accessed on 22 June 2016) [21]. This includes 60,801 CHD case and 123,504 control participants. We first searched the CARDIoGRAMplusC4D 1000 Genomes-based GWAS, a meta-analysis of GWAS studies of mainly European, South Asian and East Asian descent imputed using the 1000 Genomes phase $1 \mathrm{v} 3$ training set with 38 million variants [22]. If no summary data on the gene-CHD association were found from the 1000 Genomes data, then we screened in CARDIoGRAMplusC4D Metabochip. If the targeted SNPs were not found in either the 1000 Genomes or the CARDIoGRAMplusC4D Metabochip, we then screened CARDIoGRAM GWAS. effect (if the horizontal pleiotropic paths are in the same direction as that of the main risk factor of interest) or a diminution of the true effect (if the horizontal pleiotropic effect is in the opposite direction of the risk factor of interest). There are a number of different statistical methods that can be used to estimate causal MR effects. Many of these are related to the ratio, which is intuitive. If the assumptions above are correct then the causal effect of the risk factor (BMI) on outcome (CHD) is the ratio of 'the association of genetic instruments with CHD' to 'the association of genetic instrument with BMI'. Valid MR estimates can be obtained using two (independent) samples for the association of the genetic instrument with outcome and the association of genetic instrument with risk factor [16]. There are some advantages of this two-sample MR approach over the one-sample approach (where both parts of the ratio are obtained from the same sample), including the potential to gain very large sample sizes by using publicly available aggregate genome-wide data as we have done here and apply novel methods for testing horizontal pleiotropy that have been developed for use in two-sample MR with aggregate GWAS data (see the Methods section and the ESM for detailed descriptions of these)

The genetic variants used as instrumental variables for CHD, BMI and CHD risk factors (potential mediators) are all shown in ESM Tables 1-9.

\section{Statistical analysis}

As an indication of the strength of the association between genetic instruments and phenotypes, we report the proportion of variation in BMI and all mediators explained by their genetic variant instruments and also the F-statistic for the regression of BMI and all mediators on their genetic instruments. The proportion of the BMI-CHD effect that is explained by a group of mediators will be estimated with bias if the mediators are related to each other, and/or if the outcome has an effect on the mediator (i.e. there is reverse causality) and the instrument affects the mediators through the outcome. Therefore, we tested for potential bi-directional causal effects of BMI, potential mediators and CHD with each other using the inverse variance weighted (IVW) approach described below.

Horizontal pleiotropy, where the genetic variant influences the outcome through a pathway other than the exposure, violates an assumption of MR and can bias causal estimates. To guard against this we used three different analytical approaches for both step one (effect of BMI on CHD and potential mediators) and step two (effect of potential mediators on CHD) of the two-step MR mediation approach. 
Each of the three methods assumes different models of horizontal pleiotropy. The value of comparing results from all three is that we have more confidence in results that are consistent across the different methods. Full details of these approaches, including their different assumptions, are provided in Table 1 and ESM Methods 1.

To estimate the effect of BMI on CHD taking account of genetically determined potential mediators, we used the IVW MR method, adjusting for the SNP-potential mediator effect [23]. The proportion of the effect that is mediated by any of the potential mediators was estimated by the changes in the total effect of the genetically determined BMI on CHD risk (for more details see ESM Methods 2). This method assumes that mediators are continuously measured variables and as type 2 diabetes is dichotomised we did not assess the proportion of the BMI-CHD effect due to type 2 diabetes. An analysis diagram is shown in Fig. 2. All statistical analysis was performed using STATA 13.1 (Stata Corp LP, College Station, TX, USA) and R (version 3.2.5, the R Foundation for statistical Computing, Vienna, Austria) software.

\section{Results}

The proportion of variation explained by all of the variants that we used as instrumental variables for the potential mediators varied from $1.2 \%$ (for fasting insulin) to $5.7 \%$ (for type 2 diabetes) (ESM Tables 1-8). The first stage F-statistic for all of the MR analyses (i.e. for the regression of BMI and each of the mediators on their genetic variant instrument variables) were very large (>500).

\section{Relationships between potential mediators and CHD}

As expected, we observed evidence for association between fasting plasma glucose and type 2 diabetes, and that both fasting plasma glucose and type 2 diabetes were associated with $\mathrm{HbA}_{1 \mathrm{c}}$ (Table 2). LDL-cholesterol, HDL-cholesterol and triacylglycerols were associated with each other. CHD appears to be causally positively related to type 2 diabetes, but was not related to other potential mediators (Table 2).

\section{Effects of BMI on CHD and glycaemic and lipid traits}

There was consistent support across all three MR methods for a causal effect of higher BMI on higher CHD and type 2 diabetes risk, and higher levels of fasting glucose, $\mathrm{HbA}_{1 \mathrm{c}}$, fasting insulin and triacylglycerols, together with lower HDL-cholesterol (Table 3). None of the methods supported a causal effect of BMI on LDL-cholesterol (Table 3 and ESM Table 10).

\section{Effects of potential mediators on CHD}

There was broadly consistent support across all three MR methods for a positive effect of type 2 diabetes, $\mathrm{HbA}_{1 \mathrm{c}}$,

Table 1 Summary of the three methods used for MR analysis




Fig. 2 Analysis diagram. Summary data for SNP GWAS consortia datasets (GIANT, CARDIoGRAM, C4D, DIAGRAM, MAGIC and GLGC). MR estimates of BMI on mediators (type 2 diabetes [T2DM], fasting glucose [FG], fasting insulin [FI], $\mathrm{HbA}_{1 \mathrm{c}}$, LDLcholesterol [LDL-C], HDLcholesterol [HDL-C] and triacylglycerols [TG]), and of BMI and mediators on CHD were derived using the IVW method phenotypes were extracted from

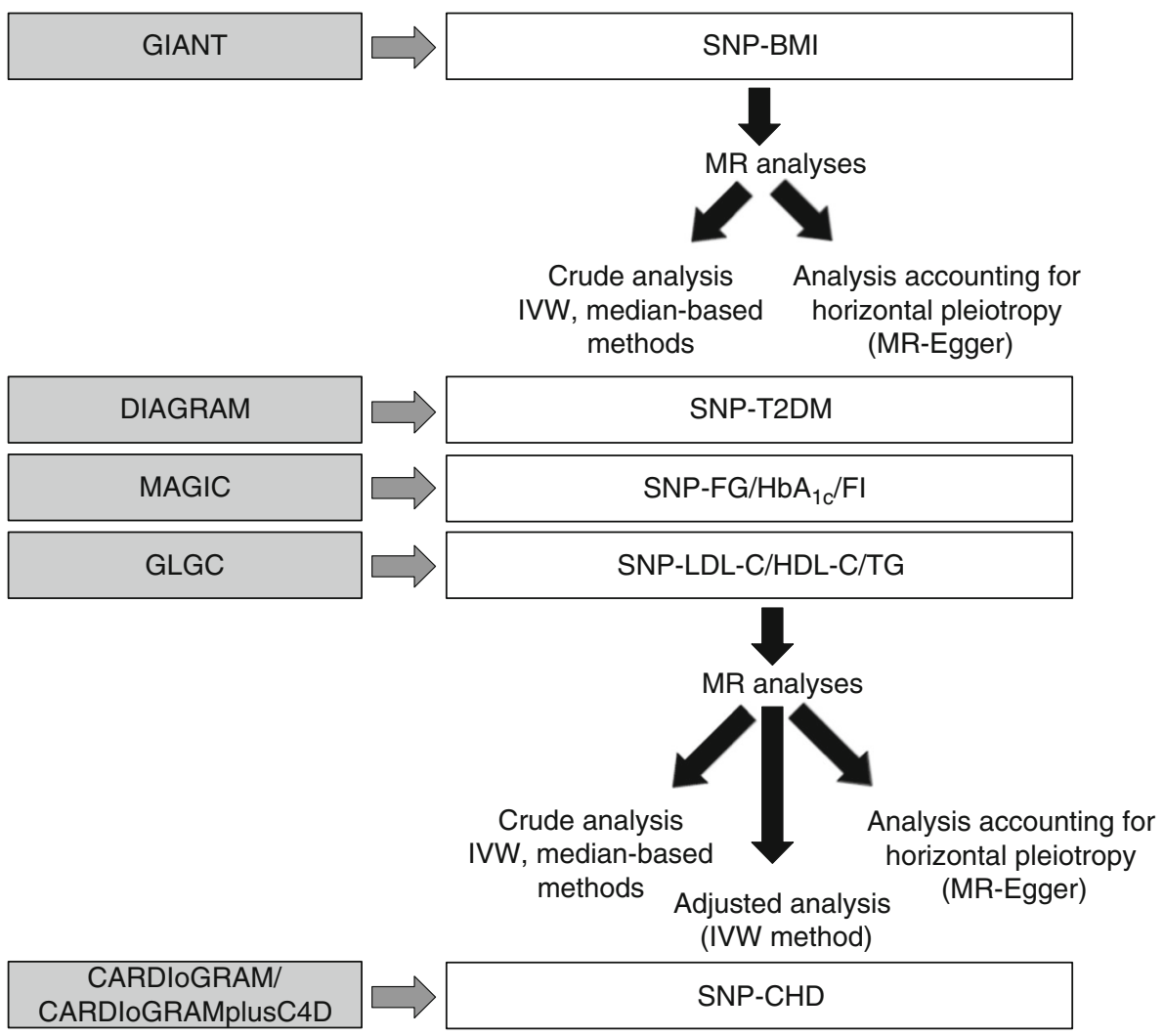

triacylglycerols and LDL-cholesterol on CHD risk (Table 4). For type 2 diabetes the MR-Egger 95\% CI just included the null value, but this method has lower statistical power than the others and the point estimates were similar across all methods. For triacylglycerols the estimate of effect (slope) from MR-Egger was a little weaker than for all of the other methods (e.g. 1.24 vs 1.13 comparing the IVW and MR-Egger methods), suggesting that some but not all of the effect of triacylglycerols estimated by IVW and other methods might be due to horizontal pleiotropy. In IVW and the median method analyses, there is evidence for the causal association of lower HDL-cholesterol and higher fasting glucose and insulin with higher risk of CHD. However, for all of these MR-Egger suggested that effects were largely due to horizontal pleiotropy, with effect estimates markedly attenuated to the null and the intercepts all being non-zero.

\section{Mediating effects of lipids and glycaemic traits on BMI-CHD effects}

We explored those potential mediators that had causal support from MR for both an effect of BMI on them (step one) and of the mediators on CHD (step two): type 2 diabetes, $\mathrm{HbA}_{1 \mathrm{c}}$ and triacylglycerols (Table 5). Our results suggested that triacylglycerols were an important mediator, with either type 2 diabetes or $\mathrm{HbA}_{1 \mathrm{c}}$ further contributing to mediation of $\mathrm{BMI}$ on CHD. The BMI-CHD effect reduced from 1.45 (95\% CI
$1.27,1.66)$ to $1.16(95 \%$ CI $0.99,1.36)$ and $1.36(95 \%$ CI $1.19,1.57)$ with adjustment for the estimated effects of triacylglycerols and $\mathrm{HbA}_{1 \mathrm{c}}$, respectively, and to 1.09 (95\% CI 0.94, 1.27) with adjustment for both.

\section{Discussion}

This is the first paper to explore the extent to which glycaemic traits mediate a causal path between BMI and CHD, whilst using statistical approaches that account for horizontal pleiotropy. Consistent with previous studies [11-14], but using a larger sample size and more genetic variants, we show that higher BMI causes greater CHD risk. Our results also suggest that triacylglycerols, $\mathrm{HbA}_{1 \mathrm{c}}$ and type 2 diabetes play important roles in causally mediating the effect of BMI on CHD. In contrast, our results do not support causal effects for the observed association between BMI and LDL-cholesterol, or for the associations of HDL-cholesterol, fasting glucose or insulin with CHD. Secondary prevention, which aims to reduce obesity-related CHD by targeting causal mediators is important because of the large, and increasing, proportions of people globally who are overweight or obese and the lack, currently, of scalable effective treatments for obesity. Treating causal mediators of the effect of BMI on CHD could mitigate its effect, but biases in conventional epidemiological methods for testing mediation have limited 


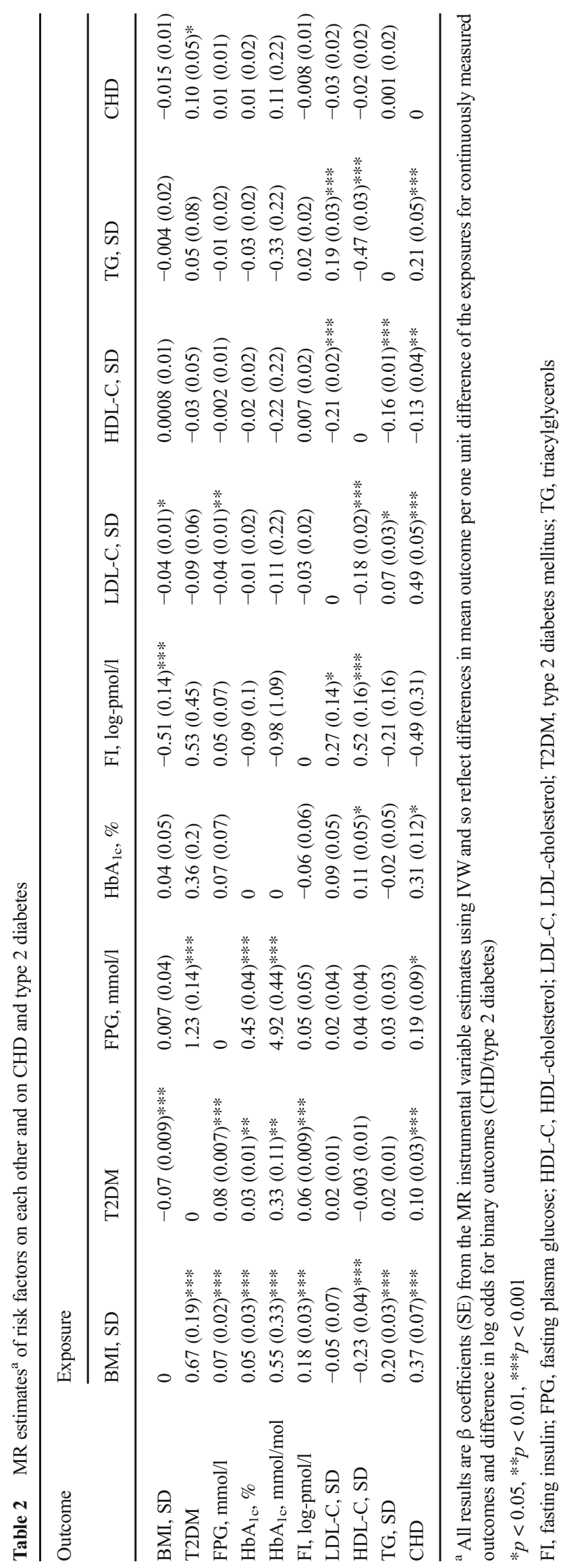


Table 3 MR estimates of BMI (SD, $\left.1 \mathrm{SD}=4.5 \mathrm{~kg} / \mathrm{m}^{2}\right)$ on cardiovascular risk factors and $\mathrm{CHD}$

\begin{tabular}{|c|c|c|c|}
\hline Exposure: BMI $(n=322,154)$ & $\begin{array}{l}\text { Effect } \\
\text { estimate }\end{array}$ & $95 \% \mathrm{CI}$ & $p$ value \\
\hline
\end{tabular}

\begin{tabular}{|c|c|c|c|}
\hline \multicolumn{4}{|c|}{ CHD $(n=60,801 \text { case and } 123,504 \text { control participants })^{\mathrm{a}}$} \\
\hline IVW & 1.45 & $1.27,1.66$ & $<0.001$ \\
\hline Weighted-median & 1.44 & $1.24,1.67$ & $<0.001$ \\
\hline \multicolumn{4}{|l|}{ MR-Egger regression } \\
\hline Slope & 1.55 & $1.26,1.91$ & $<0.001$ \\
\hline $\begin{array}{l}\text { Intercept (directional } \\
\text { pleiotropy) }\end{array}$ & 1.00 & $0.99,1.00$ & 0.50 \\
\hline \multicolumn{4}{|c|}{$\begin{array}{l}\text { Type } 2 \text { diabetes mellitus }(n=34,840 \text { case and } 114,981 \text { control } \\
\text { participants })^{\mathrm{a}}\end{array}$} \\
\hline IVW & 1.96 & $1.35,2.83$ & $<0.001$ \\
\hline Weighted-median & 2.63 & $2.16,3.21$ & $<0.001$ \\
\hline \multicolumn{4}{|l|}{ MR-Egger regression } \\
\hline Slope & 3.42 & $2.63,4.46$ & $<0.001$ \\
\hline $\begin{array}{l}\text { Intercept (directional } \\
\text { pleiotropy) }\end{array}$ & 0.98 & $0.98,0.99$ & $<0.001$ \\
\hline \multicolumn{4}{|c|}{ Fasting glucose, $\mathrm{mmol} / \mathrm{l}(n=46,186)^{\mathrm{b}}$} \\
\hline IVW & 0.07 & $0.03,0.11$ & $<0.001$ \\
\hline Weighted-median & 0.08 & $0.05,0.12$ & $<0.001$ \\
\hline \multicolumn{4}{|l|}{ MR-Egger regression } \\
\hline Slope & 0.09 & $0.036,0.15$ & $<0.001$ \\
\hline Intercept & -0.0007 & $-0.002,0.001$ & 0.37 \\
\hline \multicolumn{4}{|l|}{$\mathrm{HbA}_{1 \mathrm{c}}, \%(n=46,368)^{\mathrm{b}}$} \\
\hline IVW & 0.05 & $0.01,0.08$ & 0.005 \\
\hline Weighted-median & 0.09 & $0.04,0.14$ & $<0.001$ \\
\hline \multicolumn{4}{|l|}{ MR-Egger regression } \\
\hline Slope & 0.09 & $0.008,0.16$ & 0.03 \\
\hline Intercept & -0.001 & $-0.003,0.001$ & 0.31 \\
\hline
\end{tabular}

Fasting insulin, log-pmol/1 $(n=38,238)^{\mathrm{b}}$

\begin{tabular}{|c|c|c|c|}
\hline IVW & 0.18 & $0.14,0.22$ & $<0.001$ \\
\hline Weighted-median & 0.18 & $0.12,0.24$ & $<0.001$ \\
\hline \multicolumn{4}{|c|}{ MR-Egger regression } \\
\hline Slope & 0.16 & $0.07,0.25$ & $<0.001$ \\
\hline Intercept & 0.0007 & $-0.002,0.003$ & 0.60 \\
\hline \multicolumn{4}{|c|}{ CDL-cholesterol, SD (1 SD = $1.0 \mathrm{mmol} / \mathrm{l})(n=188,577)^{\mathrm{b}}$} \\
\hline IVW & -0.05 & $-0.19,0.09$ & 0.50 \\
\hline Weighted-median & -0.01 & $-0.08,0.05$ & 0.66 \\
\hline \multicolumn{4}{|c|}{ MR-Egger regression } \\
\hline Slope & -0.10 & $\begin{array}{r}-0.184 \\
-0.02\end{array}$ & 0.02 \\
\hline Intercept & 0.0016 & $-0.001,0.004$ & 0.19 \\
\hline \multicolumn{4}{|c|}{ HDL-cholesterol, $\mathrm{SD}(1 \mathrm{SD}=0.40 \mathrm{mmol} / \mathrm{l})(n=188,577)^{\mathrm{b}}$} \\
\hline IVW & -0.23 & $-0.32,-0.15$ & $<0.001$ \\
\hline Weighted-median & -0.21 & $-0.27,-0.16$ & $<0.001$ \\
\hline \multicolumn{4}{|c|}{ MR-Egger regression } \\
\hline Slope & -0.23 & $-0.307,-0.15$ & $<0.001$ \\
\hline Intercept & -0.0001 & $-0.002,0.002$ & 0.90 \\
\hline
\end{tabular}

Table 3 (continued)

\begin{tabular}{lccc}
\hline Exposure: BMI $(n=322,154)$ & $\begin{array}{l}\text { Effect } \\
\text { estimate }\end{array}$ & 95\% CI & $p$ value \\
\hline Triacylglycerol, SD $(1 \mathrm{SD}=1.024 \mathrm{mmol} / \mathrm{l})$ & $(n=188,577)^{\mathrm{b}}$ & \\
$\quad$ IVW & 0.20 & $0.14,0.26$ & $<0.001$ \\
$\quad$ Weighted-median & 0.21 & $0.15,0.27$ & $<0.001$ \\
MR-Egger regression & & & \\
$\quad$ Slope & 0.17 & $0.09,0.24$ & $<0.001$ \\
$\quad$ Intercept & 0.001 & $-0.001,0.003$ & 0.37 \\
\hline
\end{tabular}

${ }^{\text {a }}$ Binary outcome - effect estimate is the OR for a $1 \mathrm{SD}$ increase in BMI

${ }^{\mathrm{b}}$ Continuously measured outcome - effect estimate is the difference in mean in the unit provided in column 1 for a 1 SD increase in BMI

our understanding of which CHD risk factors mediate BMI effects. Our findings provide strong support for undertaking RCTs in obese people to test the effect of triacylglycerol reduction and glycaemic control on CHD risk.

Several MR studies have previously examined the association of BMI with CHD and CHD risk factors [11, 12, $14,24,25]$. Our results are broadly consistent with those, including our finding of no evidence for the causal association between BMI and LDL-cholesterol [12, 24, 26]. This is further supported by two RCTs of bariatric surgery which found that intensive weight loss did not lower LDL-cholesterol [27, 28]. Consistent with our results, previous MR studies have also shown positive causal effects of type 2 diabetes, $\mathrm{HbA}_{1 \mathrm{c}}$, LDL-cholesterol and triacylglycerols with CHD [29-32], but not for causal effects of fasting glucose or HDL-cholesterol with CHD once horizontal pleiotropy has been accounted for. The discrepancy between finding a causal effect of type 2 diabetes and $\mathrm{HbA}_{1 \mathrm{c}}$ on CHD, but not of fasting glucose, might suggest that non-fasting (postprandial) glucose levels, more so than fasting levels, are most relevant for CHD risk and/or that long-term hyperglycaemia (as assessed by elevated $\mathrm{HbA}_{1 \mathrm{c}}$ and likely to be identified as being above the threshold required to diagnose type 2 diabetes) are important.

To our knowledge only one previous study has tried to explore potential mediation of the BMI-CHD effect in an MR framework. That study included 11,056 individuals with CHD and 75,627 control participants from Copenhagen and used only three BMI-related SNPs. It concluded that the effect of BMI on increased CHD risk was partly mediated through elevated levels of LDL-cholesterol, non-fasting remnant cholesterol and systolic BP [14]. The evidence for a mediating role of remnant cholesterol is entirely consistent with our findings here for triacylglycerols, as remnant cholesterol is the cholesterol content of triacylglycerol-rich lipoproteins, particularly so in this previous study where remnant cholesterol was not directly measured but estimated from other lipids using 
Table 4 MR estimates of cardiovascular risk factors on CHD

\begin{tabular}{|c|c|c|c|}
\hline Risk factor & OR & $95 \% \mathrm{CI}$ & $p$ value \\
\hline \multicolumn{4}{|c|}{ Type 2 diabetes mellitus } \\
\hline IVW & 1.12 & $1.06,1.18$ & $<0.001$ \\
\hline Weighted-median & 1.11 & $1.05,1.17$ & $<0.001$ \\
\hline \multicolumn{4}{|c|}{ MR-Egger regression } \\
\hline Slope & 1.07 & $0.99,1.15$ & 0.10 \\
\hline Intercept & 1.01 & $1.00,1.01$ & 0.17 \\
\hline \multicolumn{4}{|c|}{ Fasting glucose, mmol/1 } \\
\hline IVW & 1.31 & $1.09,1.58$ & $<0.001$ \\
\hline Weighted-median & 1.21 & $1.01,1.44$ & 0.03 \\
\hline \multicolumn{4}{|c|}{ MR-Egger regression } \\
\hline Slope & 1.08 & $0.87,1.35$ & 0.50 \\
\hline Intercept & 1.01 & $1.00,1.01$ & 0.04 \\
\hline \multicolumn{4}{|l|}{$\mathrm{HbA}_{1 \mathrm{c}}, \%$} \\
\hline IVW & 1.30 & $1.08,1.56$ & 0.01 \\
\hline Weighted-median & 1.36 & $1.07,1.74$ & 0.01 \\
\hline \multicolumn{4}{|c|}{ MR-Egger regression } \\
\hline Slope & 1.66 & $1.03,2.68$ & 0.04 \\
\hline Intercept & 0.99 & $0.97,1.01$ & 0.27 \\
\hline \multicolumn{4}{|c|}{ Fasting insulin, log-pmol/1 } \\
\hline IVW & 2.80 & $1.89,4.16$ & $<0.001$ \\
\hline Weighted-median & 2.61 & $1.61,4.23$ & $<0.001$ \\
\hline \multicolumn{4}{|c|}{ MR-Egger regression } \\
\hline Slope & 0.49 & $0.09,2.59$ & 0.40 \\
\hline Intercept & 1.03 & $1.00,1.05$ & 0.04 \\
\hline \multicolumn{4}{|c|}{ LDL-cholesterol, SD (1 SD = $1.0 \mathrm{mmol} / \mathrm{l})$} \\
\hline IVW & 1.58 & $1.43,1.75$ & $<0.001$ \\
\hline Weighted-median & 1.63 & $1.48,1.80$ & $<0.001$ \\
\hline \multicolumn{4}{|c|}{ MR-Egger regression } \\
\hline Slope & 1.74 & $1.59,1.90$ & $<0.001$ \\
\hline Intercept & 0.99 & $0.98,0.99$ & 0.01 \\
\hline \multicolumn{4}{|c|}{ HDL-cholesterol, SD (1 SD = $0.4 \mathrm{mmol} / \mathrm{l})$} \\
\hline IVW & 0.86 & $0.78,0.95$ & $<0.001$ \\
\hline Weighted-median & 0.88 & $0.81,0.95$ & $<0.001$ \\
\hline \multicolumn{4}{|c|}{ MR-Egger regression } \\
\hline Slope & 1.03 & $0.95,1.12$ & 0.46 \\
\hline Intercept & 0.99 & $0.98,0.99$ & $<0.001$ \\
\hline \multicolumn{4}{|c|}{ Triacylglycerol, SD $(1 \mathrm{SD}=1.024 \mathrm{mmol} / \mathrm{l})$} \\
\hline IVW & 1.24 & $1.10,1.41$ & $<0.001$ \\
\hline Weighted-median & 1.23 & $1.11,1.36$ & $<0.001$ \\
\hline \multicolumn{4}{|c|}{ MR-Egger regression } \\
\hline Slope & 1.13 & $1.03,1.24$ & 0.01 \\
\hline Intercept & 1.01 & $1.003,1.01$ & $<0.001$ \\
\hline
\end{tabular}

a method that would produce an extremely high correlation between (measured) triacylglycerols and estimated remnant cholesterol; mediation by remnant cholesterol is thus consistent with mediation by triacylglycerols [14].
Table 5 Multivariate separate-sample MR analysis of the effect of BMI (per SD, $1 \mathrm{SD}=4.5 \mathrm{~kg} / \mathrm{m}^{2}$ ) on $\mathrm{CHD}$

\begin{tabular}{lllll}
\hline & OR & $95 \%$ CI & $p$ value & $\begin{array}{c}\text { Mediation } \\
\text { effect (\%) }\end{array}$ \\
\hline $\begin{array}{l}\text { MR-IVW regression, crude } \\
\text { Multivariate model }\end{array}$ & 1.45 & $1.27,1.66$ & $<0.001$ & \\
$\begin{array}{l}\text { (1) Adjusted for } \\
\text { triacylglycerol }\end{array}$ & 1.16 & $0.99,1.36$ & 0.06 & 22 \\
$\begin{array}{l}\text { (2) Adjusted for HbA } \mathrm{Hc}_{\mathrm{c}} \\
\text { (3) Adjusted for type 2 } \\
\text { diabetes }\end{array}$ & 1.36 & $1.19,1.57$ & 0.001 & 4 \\
$\begin{array}{l}\text { (4) Adjusted for } \\
\text { triacylglycerol + }\end{array}$ & 1.09 & $0.94,1.27$ & 0.25 & 38 \\
$\begin{array}{l}\text { HbA } \\
\text { (5) Adjusted for }\end{array}$ & 1.10 & $0.94,1.29$ & 0.22 & - \\
$\begin{array}{l}\text { triacylglycerol + type 2 } \\
\text { diabetes }\end{array}$ & & & & \\
\hline
\end{tabular}

Our study, the previous (Copenhagen) study [13,14], other MR studies [12, 24, 26] and RCTs of bariatric surgery [33, 34] have found no evidence for a causal effect of BMI on LDL-cholesterol, which suggests it is unlikely to be an important mediator of BMI on CHD. However, since the previous study (despite finding no MR evidence for a causal effect of BMI on LDL-cholesterol) concluded that LDL-cholesterol was a partial mediator [14], we examined that possibility in our data. As expected we found no strong support for a mediating effect of LDL-cholesterol between BMI and CHD (ESM Table 12). We were unable to explore any mediating effect of BP in our study. This is because our approach uses publicly available aggregate genome-wide results and the International Consortium for Blood Pressure (ICBP) provides information on SNPs and BP associations without specifying the risk (or effect) allele for each SNP; thus the effect of BMI on BP cannot be assessed using the two-sample MR instrumental variable analysis.

CHD is a major cause of morbidity and mortality and its prevalence is increasing worldwide, partly because of the increasing prevalence of obesity. Our results indicate the extent to which acting on risk factors, such as triacylglycerols, $\mathrm{HbA}_{1 \mathrm{c}}$ and type 2 diabetes, might counteract the detrimental effects of obesity on CHD. They highlight the potential importance of using interventions that lower triacylglycerols and/or $\mathrm{HbA}_{1 \mathrm{c}}$ and type 2 diabetes specifically in those with obesity $[35,36]$. There is evidence, including from MR studies, that statins affect triacylglycerols and remnant cholesterol, as well as LDL-cholesterol [37]. Furthermore, a rare variant in $A P O C 3$ with a marked effect on triacylglycerol levels provides a potential target for drug development aimed at reducing triacylglycerol levels, independent of any statin effects $[38,39]$. Thus, targets for reducing triacylglycerols exist and testing the effect of these in obese populations would be feasible. Previous RCTs have shown that the oral 
hypoglycaemic metformin reduces cardiovascular risk factors [40-42] in non-diabetic at-risk populations, including those who are obese, but its effect on CHD risk has yet to be established. Our results suggest that it might be cardioprotective in populations with high BMI and supports the development of RCTs to test its effect on CHD in these people.

\section{Strengths and limitations}

Two-sample MR exploits the fact that it is not necessary to obtain both gene-exposure association (ratio denominator) and gene-outcome association (ratio numerator) from the same sample of participants. There are some advantages to obtaining them from two different sets of participants. For example, 'winners' curse' [16] can bias true causal effects towards observational results in one-sample MR but is less likely to generate false-positive findings in two-sample MR. In addition, the weak instrument bias, which biases effects towards the confounded multivariable regression result in one-sample MR, biases the effect towards the null in two-sample MR (with non-overlapping datasets). The main advantage of two-sample MR is the increased statistical power, particularly in relation to testing effects on binary disease outcomes (i.e. CHD or type 2 diabetes) because of the use of summary data from GWAS consortia [16].

Our study is extremely large and uses genetic variants to avoid some of the key limitations of traditional multivariable regression approaches to mediation. Horizontal pleiotropy is one of the major concerns in relation to limitations of MR studies. However, to explore the potential effects of this pleiotropy, we used different MR methods (IVW, medianbased estimators and MR-Egger) that have different assumptions and we assessed the consistency across each of these estimators. The mediators that we took forward into MR-based mediation analyses (triacylglycerols, $\mathrm{HbA}_{1 \mathrm{c}}$ and type 2 diabetes) had consistent causal effects across these different methods for both steps (i.e. the effect of BMI on them and of them on CHD). In the mediation analyses, where we include both genetically predicted triacylglycerols and $\mathrm{HbA}_{1 \mathrm{c}}$, we are assuming that these factors are not causally related to each other. We tested for causal relationships between potential mediators prior to our main two-step MR analyses and these do not suggest any causal effects between triacylglycerols and $\mathrm{HbA}_{1 \mathrm{c}}$ or other glycaemic traits. However, MR studies cannot completely rule out a causal relationship between the two. Previous large prospective studies showed triacylglycerols predicted the development of type 2 diabetes [43, 44], if this association is casual, the estimated mediation effect by dysglycaemia and triacylglycerols could be inflated. Our results would be biased if the mediators we have tested caused variation in BMI (i.e. there was reverse causality from mediators to BMI). If this were the case, we would expect a bi-directional MR effect between BMI and the mediating risk factors. However, we found no evidence that triacylglycerols or $\mathrm{HbA}_{1 \mathrm{c}}$ caused variation in BMI (though the causal effect of BMI to these mediators was present).

Whilst all three MR methods suggest a casual effect of triacylglycerols on CHD, the MR-Egger intercept suggests that directional horizontal pleiotropy may be exhibited by the instruments. It is plausible that the genetic variants we used as instruments for triacylglycerols also affect other remnant cholesterols or other lipids and those also contribute to mediating BMI effects on CHD. Another potential limitation to our study is that we have assumed no interaction between BMI and mediators, but we are not able to test for this because we have used aggregated genome-wide data. Previous observational studies suggest that the association between BMI and CHD may be modified by hypertension [45], but have not found effect modification by the glycaemic and lipid traits that we have examined here [46]. In twosample MR, with independent samples, weak instrument bias can result in bias towards the null. In mediation analyses this could result in an underestimation of mediating effects. However, given our large sample size and the fact that our genetic instruments explained $2.1 \%$ and $2.4 \%$ of the variation in triacylglycerols and $\mathrm{HbA}_{1 \mathrm{c}}$, respectively, and had very large first stage F-statistics, we think this is unlikely to have had a major effect on our results. In addition, there is a partial overlap in studies that contributed to both GWAS (i.e. some cohort studies have contributed both to GWAS of exposure and also of outcomes). Of the 38 studies included in the CARDIoGRAMplusC4D, 24 appear in GIANT (about 30\% of participant overlap) [47]. In the case of weak instruments, the sample overlap between the exposure- and outcomeconsortia could bias two-sample MR estimates towards the confounded association between the exposure and the outcome $[16,47]$. Nevertheless, as we used genetic instruments strongly associated with our exposure, as suggested by large F-statistics, it is unlikely that our results were biased by weak instruments [47].

In conclusion, our results support a causal effect of higher $\mathrm{BMI}$ on CHD risk that is, at least partially, mediated through the effect of $\mathrm{BMI}$ on triacylglycerols, $\mathrm{HbA}_{1 \mathrm{c}}$ and type 2 diabetes. These findings support the need for interventional studies examining whether lowering triacylglycerols or providing glucose-lowering therapy for people who are overweight or obese is effective at reducing their increased risk (in comparison with people of healthy weight) of CHD.

Data availability Data on BMI were contributed by the GIANT consortium and downloaded from http://www.broadinstitute.org/ collaboration/giant/index.php/GIANT_consortium_data_files. Data on coronary artery disease/myocardial infarction were contributed by CARDIoGRAMplusC4D investigators and downloaded from www. 
CARDIOGRAMPLUSC4D.ORG. Data on lipid traits were contributed by GLGC and downloaded from http://csg.sph.umich.edu/abecasis/ public/lipids2013/. Data on glycaemic traits were contributed by MAGIC investigators and downloaded from www.magicinvestigators. org. All of these data are publicly available. Investigators who have made their genome-wide data available may not necessarily agree with comments made in this article and the authors take full responsibility for the content of this article.

Funding The research leading to these results has received funding from the European Research Council under the European Union's Seventh Framework Programme (FP/2007-2013) / ERC Grant Agreement (Grant number 669545; DevelopObese) and from the European Union's Horizon 2020 research and innovation programme under grant agreement No 733206 (LifeCycle). LX receives financial support from the University of Hong Kong/China Medical Board (CMB 2015/16 First Round). MCB received financial support from Conselho Nacional de Desenvolvimento Científico e Tecnológico (CNPq) (fellowship number 144749/2014-9, 201498/2014-6 [Science Without Borders Programme]) and Coordenação de Aperfeiçoamento de Pessoal de Nível Superior (CAPES) (fellowship number 201498/ 2014-6). GH, MCB and DAL work in a unit that receives funding from the UK Medical Research Council (MC UU 12013/5) and DAL is a UK National Institute of Health Research Senior Investigator (NF-SI-061110196). The funding bodies had no role in study design, data collection and analysis, decision to publish or preparation of the manuscript. The work is of the authors and the views expressed here may not be the views of any funding bodies.

Duality of interest DAL has received support from Roche Diagnostic and Medtronic in relation to some of her biomarker research that is not related to the research presented here. The other authors declare that they have no conflicts of interest (LX, MCB, GH).

Contribution statement DAL, LX, MCB and GH have made substantial contributions to study conception and design, acquisition of data, analysis and interpretation of data, and drafting or revising the article critically for important intellectual content, and provided final approval of the version to be published. DAL and LX are responsible for the integrity of the work as a whole.

Open Access This article is distributed under the terms of the Creative Commons Attribution 4.0 International License (http:// creativecommons.org/licenses/by/4.0/), which permits unrestricted use, distribution, and reproduction in any medium, provided you give appropriate credit to the original author(s) and the source, provide a link to the Creative Commons license, and indicate if changes were made.

\section{References}

1. Courcoulas AP, Christian NJ, Belle SH et al (2013) Weight change and health outcomes at 3 years after bariatric surgery among individuals with severe obesity. JAMA 310:2416-2425

2. Cholesterol Treatment Trialists Collaborators (2012) The effects of lowering LDL cholesterol with statin therapy in people at low risk of vascular disease: meta-analysis of individual data from 27 randomised trials. Lancet 380:581-590

3. Gerstein HC, Miller ME, Ismail-Beigi F et al (2014) Effects of intensive glycaemic control on ischaemic heart disease: analysis of data from the randomised, controlled ACCORD trial. Lancet 384:1936-1941

4. Baron RM, Kenny DA (1986) The moderator-mediator variable distinction in social psychological research: conceptual, strategic, and statistical considerations. J Pers Soc Psychol 51:1173-1182

5. Richmond RC, Hemani G, Tilling K, Davey Smith G, Relton CL (2016) Challenges and novel approaches for investigating molecular mediation. Hum Mol Genet 25:R149-R156

6. Hemani G, Tilling K, Davey Smith G (2017) Orienting the causal relationship between imprecisely measured traits using genetic instruments. bioRxiv. doi:10.1101/117101

7. Smith GD, Lawlor DA, Harbord R, Timpson N, Day I, Ebrahim S (2007) Clustered environments and randomized genes: a fundamental distinction between conventional and genetic epidemiology. PLoS Med 4:e352

8. Lawlor DA, Harbord RM, Sterne JA, Timpson N, Davey Smith G (2008) Mendelian randomization: using genes as instruments for making causal inferences in epidemiology. Stat Med 27:1133-1163

9. Ference BA, Majeed F, Penumetcha R, Flack JM, Brook RD (2015) Effect of naturally random allocation to lower low-density lipoprotein cholesterol on the risk of coronary heart disease mediated by polymorphisms in NPC1L1, HMGCR, or both: a 2 × 2 factorial Mendelian randomization study. J Am Coll Cardiol 65:1552-1561

10. Ference BA, Julius S, Mahajan N, Levy PD, Williams KA Sr, Flack JM (2014) Clinical effect of naturally random allocation to lower systolic blood pressure beginning before the development of hypertension. Hypertension 63:1182-1188

11. Hagg S, Fall T, Ploner A et al (2015) Adiposity as a cause of cardiovascular disease: a Mendelian randomization study. Int J Epidemiol 44:578-586

12. Holmes MV, Lange LA, Palmer T et al (2014) Causal effects of body mass index on cardiometabolic traits and events: a Mendelian randomization analysis. Am J Hum Genet 94:198-208

13. Nordestgaard BG, Palmer TM, Benn M et al (2012) The effect of elevated body mass index on ischemic heart disease risk: causal estimates from a Mendelian randomisation approach. PLoS Med 9:e1001212

14. Varbo A, Benn M, Smith GD, Timpson NJ, Tybjaerg-Hansen A, Nordestgaard BG (2015) Remnant cholesterol, low-density lipoprotein cholesterol, and blood pressure as mediators from obesity to ischemic heart disease. Circ Res 116:665-673

15. Bowden J, Davey Smith G, Burgess S (2015) Mendelian randomization with invalid instruments: effect estimation and bias detection through egger regression. Int J Epidemiol 44:512-525

16. Lawlor DA (2016) Commentary: two-sample Mendelian randomization: opportunities and challenges. Int J Epidemiol 45:908-915

17. Locke AE, Kahali B, Berndt SI et al (2015) Genetic studies of body mass index yield new insights for obesity biology. Nature 518:197206

18. Morris AP, Voight BF, Teslovich TM et al (2012) Large-scale association analysis provides insights into the genetic architecture and pathophysiology of type 2 diabetes. Nat Genet 44:981-990

19. Dupuis J, Langenberg C, Prokopenko I et al (2010) New genetic loci implicated in fasting glucose homeostasis and their impact on type 2 diabetes risk. Nat Genet 42:105-116

20. Global Lipids Genetics Consortium (2013) Discovery and refinement of loci associated with lipid levels. Nat Genet 45:1274-1283

21. Schunkert H, Konig IR, Kathiresan S et al (2011) Large-scale association analysis identifies 13 new susceptibility loci for coronary artery disease. Nat Genet 43:333-338

22. Nikpay M, Goel A, Won HH et al (2015) A comprehensive 1,000 genomes-based genome-wide association meta-analysis of coronary artery disease. Nat Genet 47:1121-1130 
23. Burgess S, Dudbridge F, Thompson SG (2015) Re: "multivariable Mendelian randomization: the use of pleiotropic genetic variants to estimate causal effects". Am J Epidemiol 181:290-291

24. Fall T, Hagg S, Magi R et al (2013) The role of adiposity in cardiometabolic traits: a Mendelian randomization analysis. PLoS Med 10:e1001474

25. Millard LA, Davies NM, Timpson NJ, Tilling K, Flach PA, Davey Smith G (2015) MR-PheWAS: hypothesis prioritization among potential causal effects of body mass index on many outcomes, using Mendelian randomization. Sci Rep 5:16645

26. Fall T, Hagg S, Ploner A et al (2015) Age- and sex-specific causal effects of adiposity on cardiovascular risk factors. Diabetes 64: $1841-1852$

27. Martins C, Strommen M, Stavne OA, Nossum R, Marvik R, Kulseng B (2011) Bariatric surgery versus lifestyle interventions for morbid obesity-changes in body weight, risk factors and comorbidities at 1 year. Obes Surg 21:841-849

28. Herrera MF, Pantoja JP, Velazquez-Fernandez D et al (2010) Potential additional effect of omentectomy on metabolic syndrome, acute-phase reactants, and inflammatory mediators in grade III obese patients undergoing laparoscopic roux-en-Y gastric bypass: a randomized trial. Diabetes Care 33:1413-1418

29. Ahmad OS, Morris JA, Mujammami M et al (2015) A Mendelian randomization study of the effect of type-2 diabetes on coronary heart disease. Nat Commun 6:7060

30. Ross S, Gerstein HC, Eikelboom J, Anand SS, Yusuf S, Pare G (2015) Mendelian randomization analysis supports the causal role of dysglycaemia and diabetes in the risk of coronary artery disease. Eur Heart J 36:1454-1462

31. Holmes MV, Asselbergs FW, Palmer TM et al (2015) Mendelian randomization of blood lipids for coronary heart disease. Eur Heart J 36:539-550

32. Jansen H, Samani NJ, Schunkert H (2014) Mendelian randomization studies in coronary artery disease. Eur Heart J 35:1917-1924

33. Mensinger JL, Calogero RM, Stranges S, Tylka TL (2016) A weight-neutral versus weight-loss approach for health promotion in women with high BMI: a randomized-controlled trial. Appetite 105:364-374

34. The Look AHEAD Research Group (2013) Cardiovascular effects of intensive lifestyle intervention in type 2 diabetes. N Engl J Med 369:145-154

35. Miller M, Stone NJ, Ballantyne C et al (2011) Triglycerides and cardiovascular disease: a scientific statement from the American Heart Association. Circulation 123:2292-2333

36. Perk J, De Backer G, Gohlke H, et al (2012) European guidelines on cardiovascular disease prevention in clinical practice (version 2012): the fifth joint task force of the European Society of Cardiology and Other Societies on cardiovascular disease prevention in clinical practice (constituted by representatives of nine societies and by invited experts). Developed with the special contribution of the European Association for Cardiovascular Prevention \& rehabilitation (EACPR). Eur Heart J 33:1635-1701

37. Wurtz P, Wang Q, Soininen P et al (2016) Metabolomic profiling of statin use and genetic inhibition of HMG-CoA reductase. J Am Coll Cardiol 67:1200-1210

38. Timpson NJ, Walter K, Min JL et al (2014) A rare variant in APOC3 is associated with plasma triglyceride and VLDL levels in Europeans. Nat Commun 5:4871

39. Drenos F, Smith GD, Ala-Korpela M et al (2016) Metabolic characterization of a rare genetic variation within $\mathrm{APOC} 3$ and its lipoprotein lipase independent effects. Circ Cardiovasc Genet 9:231239

40. Fontbonne A, Diouf I, Baccara-Dinet M, Eschwege E, Charles MA (2009) Effects of 1-year treatment with metformin on metabolic and cardiovascular risk factors in non-diabetic upper-body obese subjects with mild glucose anomalies: a post-hoc analysis of the BIGPRO1 trial. Diabete Metab 35:385-391

41. Eppinga RN, Hartman MH, van Veldhuisen DJ et al (2016) Effect of metformin treatment on lipoprotein subfractions in non-diabetic patients with acute myocardial infarction: a glycometabolic intervention as adjunct to primary coronary intervention in ST elevation myocardial infarction (GIPS-III) trial. PLoS One 11:e145719

42. Charles MA, Morange P, Eschwege E, Andre P, Vague P, JuhanVague I (1998) Effect of weight change and metformin on fibrinolysis and the von Willebrand factor in obese nondiabetic subjects: the BIGPRO1 study. Biguanides and the prevention of the risk of obesity. Diabetes Care 21:1967-1972

43. Skretteberg PT, Grytten AN, Gjertsen K et al (2013) Triglyceridesdiabetes association in healthy middle-aged men: modified by physical fitness? A long term follow-up of 1962 Norwegian men in the Oslo ischemia study. Diabetes Res Clin Pract 101:201-209

44. Hjellvik V, Sakshaug S, Strom H (2012) Body mass index, triglycerides, glucose, and blood pressure as predictors of type 2 diabetes in a middle-aged Norwegian cohort of men and women. Clin Epidemiol 4:213-224

45. Mahamat A, Richard F, Arveiler D et al (2003) Body mass index, hypertension and 5-year coronary heart disease incidence in middle aged men: the PRIME study. J Hypertens 21:519-524

46. Prospective Studies Collaboration, Whitlock G, Lewington S, et al (2009) Body-mass index and cause-specific mortality in 900000 adults: collaborative analyses of 57 prospective studies. Lancet 373: 1083-1096

47. Burgess S, Davies NM, Thompson SG (2016) Bias due to participant overlap in two-sample Mendelian randomization. Genet Epidemiol 40:597-608 